\title{
A homocisteína como fator de risco para a aterosclerose
}

\author{
Daniel Rui Diniz-Santos ${ }^{l}$ \\ Gabriela Chagas Freitas de Andrade ${ }^{I}$
}

\begin{abstract}
Resumo
O conhecimento dos fatores de risco para doenças cardiovasculares é importante, não apenas para direcionar corretamente estratégias preventivas, como para identificar indivíduos sob risco cardiovascular, mesmo que não apresentem nenhum dos fatores de risco convencionais: diabetes, hipertensão, sedentarismo e tabagismo. Nos últimos anos, a homocisteína tem recebido grande atenção da comunidade científica, e diversos estudos indicam que esse pode ser um importante fator de risco cardiovascular, embora controvérsias ainda permaneçam. Este trabalho tem por objetivo revisar a associação entre os níveis séricos de homocisteína e o desenvolvimento da doença aterosclerótica, bem como os mecanismos pelos quais essa associação se manifesta. Com base nos artigos abordados, observa-se que os níveis plasmáticos de homocisteína têm sido freqüentemente associados ao desenvolvimento de aterosclerose e que, recentemente, têm sido obtidas evidências suficientes para sugerir que se trata de uma relação causal, direta e independente da ação de outros fatores. Entretanto, o impacto clínico de estratégias voltadas para a redução dos níveis séricos de homocisteína ainda precisa ser determinado por estudos clínicos de grande porte.
\end{abstract}

Palavras-chave: homocisteína; aterosclerose; doença cardiovascular.

\section{INTRODUÇÃO}

A aterosclerose é uma doença vascular que acomete principalmente as artérias de grande calibre (aorta, coronárias, carótidas, ilíacas e poplíteas), causando espessamento e perda da elasticidade da parede arterial. A doença aterosclerótica sintomática localiza-se mais freqüentemente nas artérias que suprem o coração, o cérebro e os rins, gerando infarto agudo do miocárdio, infarto cerebral, aneurismas de aorta e redução aguda ou crônica da perfusão arterial, além de morte súbita, cardiopatia e encefalopatia isquêmicas. A doença geralmente começa a se desenvolver ainda na infância, com a sintomatologia tornando-se evidente a partir da meia-idade, quando as lesões arteriais atingem magnitude suficiente para apresentar repercussão clínica.

A aterosclerose é uma entidade de distribuição mundial que atinge proporções epidêmicas nas sociedades economicamente desenvolvidas. Estima-se que a aterosclerose e as doenças correlatas sejam responsáveis por cerca de $50 \%$ das mortes ocorridas anualmente no hemisfério ocidental (1), o que estimula todos os campos da pesquisa biomédica a investigarem possíveis fatores de risco para o desenvolvimento da aterosclerose.

\footnotetext{
${ }^{1}$ Acadêmico do Curso de Medicina da Universidade Federal da Bahia.

Correspondência para / Correspondence to:

Daniel Rui Diniz-Santos

Av. Princesa Isabel, no 549, apto. 11 - Barra Avenida.

40130-030. Salvador - Bahia -Brasil.

Tel/Fax: (71) 3264-4424.

E-mail:danieldiniz82@ig.com.br.
} 
Os fatores de risco tradicionalmente descritos para doença cardiovascular são diabetes, tabagismo, hipertensão arterial e dislipidemia. Entretanto, pouco mais de $50 \%$ dos casos de doença cardiovascular podem ser explicados por esses fatores já conhecidos. Portanto, novos fatores de risco cardiovascular precisam ser estabelecidos, visando não só à prevenção de novos casos, como também ao controle adequado dos pacientes que já se encontram sob risco. Ao lado da proteína C-reativa, do fibrinogênio, da lipoproteína A e da infecção por Chlamydia pneumoniae, a homocisteína é o maior foco das pesquisas sobre novos fatores de risco cardiovascular. A prevalência de hiperhomocisteinemia foi estimada em 5\% da população em geral e pode chegar a até $47 \%$ entre pacientes com doença vascular aterosclerótica (2).

\section{A HOMOCISTEÍNA}

A homocisteína é um intermediário do metabolismo protéico e está envolvida na conversão da metionina, um aminoácido abundante em proteínas animais, em cisteína, sendo formada a partir da desmetilação da metionina $(2$, 3).

A homocisteína é metabolizada por duas vias: remetilação; e transulfuração.

1) O ciclo da remetilação é feito através da aquisição de um grupo metil. Essa reação é catalisada pela enzima metionina sintetase, sendo a vitamina B12 (cobalamina) um fator essencial para a ação dessa enzima. O metiltetrahidrofolato funciona como doador do radical metil nessa reação, e a enzima metilenotetrahidrofolato redutase catalisa o processo.

2) Quando há excesso de metionina ou a síntese de cisteína é estimulada, a homocisteína entra na via da transulfuração(4). Nessa via, a homocisteína é irreversivelmente sulfoconjugada à serina, para formar a cistationina, numa reação catalisada pela enzima cistationina-b-sintase, cuja ação é dependente da vitamina B6 (2). A cistationina é subseqüentemente hidrolisada para formar a cisteína, que pode ser incorporada à glutationa e posteriormente metabolizada a sulfato, sendo excretada na urina (4).
Anormalidades genéticas ou adquiridas, relacionadas às funções dessas enzimas, assim como deficiência de ácido fólico, vitamina $\mathrm{B}_{6}$ ou vitamina $B_{12}$, causam elevação nos níveis séricos de homocisteína. (2)

Aproximadamente $70 \%$ da homocisteína plasmática estão ligados à albumina; cerca de $25 \%$ combinam-se com outras moléculas de homocisteína, formando um dímero; e os 5\% restantes combinam-se com outros tióis, incluindo a cisteína na forma dissulfido ("mix homocisteína-cisteína”), ou circulam como tióis livres. (4) $\mathrm{O}$ termo homocisteinemia refere-se ao pool total circulante de homocisteína.

\section{FATORES QUE INTERFEREM NO META- BOLISMO DA HOMOCISTEÍNA}

Defeitos genéticos podem causar tanto hiperhomocisteinemia severa quanto homocisteinúria clássica (homocisteinúria congênita), causada por uma deficiência da cistationina-b-sintase, que ocorre em 1 de cada 100000 nascidos vivos e leva a um aumento de 40 vezes nas taxas de homocisteína em jejum. (4) Esse evento tem diversas implicaçooes, dentre as quais o desenvolvimento de aterosclerose prematura e eventos prematuros de aterotrombose. Freqüentemente, um evento vascular ocorre antes dos 30 anos em metade dos homozigotos não tratados. Mais raros ainda são os casos de hiperhomocisteinemia por deficiência de metileno-tetrahidrofolato redutase e deficiência de metionina sintase, devidos a desordens genéticas do metabolismo da vitamina $\mathrm{B}_{12 .}$ (4)

Os níveis sangüíneos de folato, vitamina $\mathrm{B}_{12}$ e vitamina $\mathrm{B}_{6}$ estão inversamente relacionados com a concentração plasmática de homocisteína. Indivíduos com deficiência nutricional apresentam baixas concentraçōes dessas vitaminas, o que aumenta o risco de hiperhomocisteinemia. Tem sido sugerido que aproximadamente dois terços dos casos de hiperhomocisteinemia são devidos a níveis plasmáticos inadequados de um ou mais desses co-fatores vitamínicos. (2) As concentrações séricas da homocisteína podem ser aumentadas 
pelo uso de diversas drogas e por doenças que interferem no metabolismo do folato, da vitamina $\mathrm{B}_{6}$ ou da vitamina $\mathrm{B}_{12}$. Como a maior rota de clearance da homocisteína é o metabolismo renal, a insuficiência renal crônica causa um aumento considerável dos níveis plasmáticos de homocisteína diretamente, sem interferir em nenhum desses co-fatores.

\section{DOSAGEM DA HOMOCISTEÍNA SÉRICA}

A maioria dos ensaios para a medida das concentraçôes de homocisteína é baseada em técnicas de cromatografia (5). Os níveis plasmáticos são freqüentemente medidos em jejum, e a medição pode ser feita antes e depois de uma carga de metionina. O procedimento envolve medidas basais do nível de homocisteína em jejum e uma nova medida 4 a 6 horas após a administração de uma dose oral padronizada de metionina. A carga de metionina pode ajudar a descriminar entre defeitos que envolvem a transulfuração e a remetilação, bem como ajudar a identificar pacientes com diminuição do metabolismo da metionina, apesar de apresentarem níveis plasmáticos de homocisteína normais em jejum, revelando, assim, um aumento no risco de desenvolver doença vascular.

Os estudos clínicos da homocisteína incluem as medidas plasmáticas de homocisteína, de misturas de dissulfetos que envolvem homocisteína, homocisteína tiolactona, homocisteína livre e homocisteína ligada à proteína, sendo essa última correspondente a 7080\% do total do pool. (6)

As concentrações plasmáticas normais de homocisteína em jejum estão entre 5 e 15 micromol/L.(4) Kang, Wong e Malinow classificaram os níveis de hiperhomocisteinemia em moderada (15 a 30 micromol/L), intermediária (30 a $100 \mathrm{micromol} / \mathrm{L}$ ) e severa (mais de 100 micromol/L), com essas medidas efetuadas em jeju.(7).

\section{CAUSAS DE HIPERHOMOCISTEI-NEMIA}

Vários fatores ambientais, que incluem deficiência de vitaminas, doenças sistêmicas e influência de algumas drogas, são capazes de alterar as concentraçóes plasmáticas de homocisteína.

$\mathrm{O}$ ácido fólico e a vitamina $\mathrm{B}_{12}$ são necessários para a remetilação da homocisteína, e a deficiência dessas vitaminas pode aumentar dos níveis de homocisteína, ao inibir a remetilação. A vitamina $B_{6}$ é necessária para transulfuração, e sua deficiência pode causar homocisteinemia, por aumentar os níveis de metionina. Recentemente, Santarelli e colaboradores demonstraram que baixos níveis de vitamina $B_{12}$ e gastrite atrófica aumentam significativamente o risco de hiperhomocisteinemia (odds ratio de 3,7 e 5,3, respectivamente)(8).

Dentre as possíveis causas de hiperhomocisteinemia, destacam-se, ainda, fatores fisiológicos (aumento da idade, menopausa, dieta rica em metionina), estilo de vida (tabagismo e consumo de café), herança genética (deficiência de cistationa $b$ sintase, mutação da metileno tetrahidrofolato redutase, deficiência de metionina sintase e deficiência de metileno tetrahidrofolato homocisteína metil transferase), deficiência de vitaminas $B_{12}, B_{6}$ e ácido fólico, doenças diversas (hepatopatias, insuficiência renal, diabetes melitus, lúpus eritematoso sistêmico, psoríase, anorexia nervosa, hipotireoidismo, leucemia linfoblástica aguda e transplantes) e o uso de certas drogas (anticonvulsivantes, azatioprina, diuréticos tiazídicos, ácido nicotínico e contraceptivos orais) (9).

\section{HOMOCISTEÍNA E ATEROSCLEROSE}

Há mais de 30 anos, os níveis plasmáticos de homocisteína têm sido associados ao desenvolvimento de aterosclerose (10), mas apenas recentemente foram obtidas evidências suficientes para sugerir que se trata de uma relação causal e independente da ação de outros fatores $(2,4,6)$.

Diversos estudos realizados nas últimas décadas forneceram fortes subsídios que indicam a homocisteína como um fator de risco independente para o desenvolvimento de aterosclerose $(2,4,6,9)$. Embora possa acarre- 
tar complicações vasculares sem a contribuição de outros fatores de risco, há evidência epidemiológica que mostra uma forte associação dos níveis séricos de homocisteína com alguns dos principais fatores de risco para doenças cardiovasculares, como sexo masculino, idade, tabagismo, hipercolesterolemia, hipertensão arterial e sedentarismo.(11)

A hiperhomocisteinemia está associada com doença vascular periférica (odds ratio (OR) $=1,7)$, doença cerebrovascular $(\mathrm{OR}=1,7)$, doença isquêmica do coração $(\mathrm{OR}=2,0) \mathrm{e}$ tromboembolismo venoso $(\mathrm{OR}=2,2)(9)$. $\mathrm{Um}$ incremento de $5 \mathrm{micromol} / \mathrm{L}$ de homocisteína no plasma está associado a um aumento de $60 \%$ na incidência de isquemia cardíaca, um efeito similar ao aumento de $20 \mathrm{mg} / \mathrm{dL}$ no nível sérico de colesterol. Em um estudo caso-controle, níveis elevados de homocisteína foram encontrados em $42 \%$ dos pacientes com acidente vascular cerebral e em $30 \%$ dos pacientes com doença arterial coronariana, enquanto nenhum dos controles apresentou elevação na homocisteína sérica (12).

Recentemente, Geisel e colaboradores demonstraram que a homocisteína é um eficiente marcador de risco cardiovascular, especialmente se sua dosagem for associada à dosagem da proteína C-reativa sérica: pacientes com proteína C-reativa sérica maior que $5 \mathrm{mg} / \mathrm{dL}$ e homocisteína sérica acima de 9,6 micromol/L apresentam risco significativamente maior de apresentar doença aterosclerótica coronariana que os controles $(\mathrm{OR}=12)$. Nos pacientes que apresentavam proteína $\mathrm{C}$-reativa sérica menor que $5 \mathrm{mg} / \mathrm{dL}$, a mesma elevação no risco foi encontrada com homocisteinemias superiores a 16,6 micromol/L.(13)

Estudando apenas mulheres jovens, Van den Bosch e colaboradores determinaram que a hiperhomocisteinemia sozinha aumenta o risco de doença arterial obstrutiva periférica em 2,5 vezes (14). Segundo os mesmos autores, a associação da hiperhomocisteinemia com fatores de risco tradicionais aumenta ainda mais o risco relativo, como observado nas pacientes tabagistas $(\mathrm{OR}=18,9)$, hipertensas $(\mathrm{OR}=10,3)$, diabéticas $(\mathrm{OR}=8,9)$ e dislipidêmicas $(\mathrm{OR}=8,5)$ (14).
Em uma amostra de 126 idosos com doença vascular (média de idade $=68$ anos), Spark, Laws e Fitridge observaram hiperhomocisteinemia em $33 \%$, sendo que a prevalência subia para $50 \%$ nos pacientes com angina instável e para 53\% quando se levava em conta apenas os pacientes com aneurisma de aorta (15). Nenhum dos pacientes desse estudo tinha deficiência de vitamina $\mathrm{B}_{12}$ ou de folato.

Uma meta-análise publicada por Boushey e colaboradores revelou que 34 de 38 estudos encontraram relação direta entre altos níveis séricos de homocisteína e risco aumentado de aterosclerose, sugerindo que a análise dos níveis séricos de homocisteína seja ao menos tão importante quanto a do colesterol (16).

Segundo os estudos mais recentes, a homocisteína tem uma relação direta, causal e dose-dependente com a geração de aterosclerose $(2,4,6)$. Já foi demonstrado que a incubação de hepatócitos com 4 micromol/L de homocisteína aumenta consideravelmente a síntese hepática de colesterol, ao aumentar a atividade da enzima HMG-CoA redutase, que catalisa a etapa limitante da biossíntese do colesterol (1). Uma vez que o colesterol realiza um importante papel na regulação da secreção de apoproteína (Apo) B100 pelos hepatócitos, é plausível que o aumento na síntese de colesterol e, portanto, a homocisteína, relacione-se com o um aumento na secreção da Apo B-100. A Apo B-100 é a única apoproteína presente na lipoproteína de baixa densidade (LDL), sendo responsável pela sua adesão às células e, por conseguinte, participa ativamente do processo aterosclerótico.

Guthikonda e Hayns (9) sugerem que os efeitos cardiovasculares da homocisteína podem ser explicados parcialmente através da potencialização de dois processos interligados: trombose; e aterosclerose.

1) Altas concentrações de homocisteína (300 micromol/L) podem promover o início da coagulação, através da expressão e ativação do fator tissular da célula endotelial in vitro.

2) A homocisteína, em diversas doses, está associada à aterosclerose, por exercer citotoxidade endotelial, o que não só favorece a lesão do endotélio vascular, como também diminui a 
produção de mediadores endoteliais, em especial o óxido nítrico.

Doses na ordem de $10 \mathrm{micromol} / \mathrm{L}$ lesam células endoteliais em cultura; além disso, a homocisteína inibe a síntese de DNA em células vasculares endoteliais. Por conta de suas propriedades pró-oxidadantes, a homocisteína gera peróxido de hidrogênio, que é capaz de lesar células endoteliais, e radicais superóxidos, que inibem a resposta vascular ao óxido nítrico. A homocisteína oxida a LDL e pode, posteriormente, promover captação celular de LDL oxidada, um importante passo para o processo aterosclerótico.

Segundo Buemi e colaboradores, a homocisteína promove o crescimento da musculatura lisa dos vasos sangüíneos e inibe o crescimento do endotélio vascular de maneira dosedependente. A incubação de anéis vasculares em um meio com 15 micromol/L de homocisteína aumenta as taxas de necrose em $20 \%$ e de apoptose em 18\% (17). A hiperhomocisteinemia está associada à destruição do componente elástico da parede dos vasos e à hipertrofia das células endoteliais (9), podendo promover, assim, hipertensão arterial, um importante fator de risco cardiovascular.

Os efeitos da exposição à homocisteína relacionam-se não somente ao estímulo à proliferação, mas principalmente ao desequilíbrio entre proliferação e morte celular. Tais efeitos são reduzidos pela presença do folato, que estimula a redução da concentração de homocisteína, ao favorecer seu catabolismo via remetilação em metionina, assim como ao estimular o efeito da 5'-metiltetrahidrohomocisteina-metiltransferase e da 5,10metileno-tetrahidrofolato redutase. $\mathrm{O}$ estudo de Boushey e colaboradores sugere que 50000 mortes poderiam ser evitadas anualmente nos Estados Unidos pelo aumento na ingestão de folato (16).

Os níveis de homocisteína são inversamente proporcionais aos de folato e vitamina B12, sugerindo que o risco de doença cardiovascular pode ser reduzido por suplementação vitamínica (18). Os níveis séricos de homocisteína podem ser assumidos como um indicador do estado nutricional vitamínico do corpo humano, porque refletem rapidamente alteraçôes nos níveis das vitaminas $\mathrm{B}_{6}$ e $\mathrm{B}_{12}$ e do ácido fólico: 77 de 78 pacientes com deficiência de vitamina $B_{12}$ e 18 de 19 pacientes com deficiência de folato apresentaram elevação significativa dos níveis séricos de homocisteína em relação a controles saudáveis, no estudo de Stabler e colaboradores (19).

Diversos estudos de intervenção sugerem que o tratamento com reposição vitamínica pode ter bons resultados na prevenção e no prognóstico das doenças cardiovasculares. Pietrzik e Brönstrup mostraram que a administração de $400 \mathrm{mg}$ de folato, $2 \mathrm{mg}$ de vitamina $\mathrm{B}_{6}$ e $6 \mathrm{mg}$ de vitamina $B_{12}$ por 4 semanas diminuiu os níveis séricos de homocisteína, em média $21 \%$ em relação ao grupo controle, ao qual havia sido administrado placebo (20). Embora relevantes, os dados desse estudo devem ser avaliados com certa cautela, devido à amostragem que, além de pequena $(n=72)$, era composta apenas por mulheres jovens e saudáveis.

Outro estudo de intervenção, um ensaio clínico randomizado duplo-cego controlado por placebo, realizou reposição vitamínica à base de folato e vitamina $B_{6}$ em 158 irmãos de pacientes com aterosclerose precoce. Esses indivíduos não tinham sintomas de aterosclerose, e 104 tinham hiperhomocisteinemia; os 54 restantes não tinham. Ao fim de 2 anos do estudo, constatou-se que a homocisteinemia dos indivíduos que haviam recebido vitaminas era significativamente menor do que a dos controles, tanto em jejum quanto após sobrecarga de metionina, ocorrendo o mesmo tipo de redução na ocorrência de anormalidades eletrocardiográficas durante testes de esforço (21). Está bem determinado que a suplementação vitamínica é eficaz em reduzir os níveis plasmáticos de homocisteína, mas se essa intervenção, sozinha, é capaz de reduzir o risco cardiovascular ainda não foi estabelecido.

Dos mecanismos moleculares pelos quais a homocisteína possivelmente se manifesta de forma a interferir na fisiologia vascular, o que ganha cada vez mais força na literatura é a interferência na vasodilatação, provavelmente ao afetar de várias formas a disponibilidade de óxido nítrico $(\mathrm{NO})$, um dos mais potentes vasodilata- 
dores conhecidos, que exerce papel de destaque na fisiopatologia da aterosclerose. O NO exerce um papel-chave em um dos mais importantes sistemas de regulação da função endotelial em que, além de atuar como um vasodilatador secretado pelo endotélio em resposta a estímulos diversos, inibe o crescimento da musculatura lisa vascular e a aderência plaquetária ao endotélio.

Sabe-se que a homocisteína, mesmo em doses baixas como $5 \mathrm{micromol} / \mathrm{L}$, diminui a geração de óxido nítrico por células endoteliais em cultura, alterando a vasodilatação endotéliodependente, mas não interferindo na vasodilatação endotélio-independente (22). A vasodilatação mediada por NO também pode estar reduzida devido à redução da disponibilidade do NO sintetizado, que pode ser causada pela reação espontânea do $\mathrm{NO}$ com grupos tiol da própria homocisteína, ou com o ânion superóxido $\left(\mathrm{O}_{2}^{-}\right)$que, além de ser um metabólito gerado por múltiplos processos celulares, pode ser gerado pela auto-oxidação da homocisteína, que ocorre na presença de metais (23). Além de sintetizar $\mathrm{O}_{2}^{-}$, a homocisteína também produz peróxido de hidrogênio, uma molécula nociva às células de uma maneira geral, e que também pode ser implicada no processo de disfunção endotelial induzido pela homocisteína. A homocisteína pode não só reduzir a síntese de glutation peroxidase, como também diminuir a quantidade de moléculas de RNAm que codificam essa importante enzima antioxidante no citoplasma, amplificando ainda mais o estresse oxidativo (22).

Num importante trabalho realizado in vitro, que rendeu a um de seus autores o prêmio anual do American College of Cardiology, Stühlinger e colaboradores propuseram mais um mecanismo pelo qual a homocisteína inibiria a síntese de NO (24). Como se sabe, os grupos tiol da homocisteína lhe conferem forte poder oxidante. Sobre esse dado, embasa-se a nova teoria, segundo a qual a homocisteína reage com um grupo sulfidril crítico para o bom funcionamento de uma enzima chamada dimetilarginina-dimetilaminohidrolase (DDAH).
A DDAH apresenta duas isoformas, DDAH-1 e DDAH-2, e ao menos uma delas está presente em qualquer célula do corpo humano, sendo que DDAH-2 é a isoforma encontrada nos tecidos que expressam a isoforma endotelial da enzima óxido nítrico sintetase (eNOS). A DDAH degrada a dimetilarginina assimétrica (ADMA), dando origem à citrulina e à dimetilamina. A ADMA é produzida no catabolismo de proteínas que contêm resíduos metilados de arginina. Uma vez formada, a ADMA pode ser hidrolisada e excretada na urina, ou ser catabolizada pela DDAH. Uma característica da ADMA é inibir diretamente a eNOS por competição com a L-arginina em seu sítio ativo.

Dessa forma, percebe-se que, ao inibir a DDAH, a homocisteína contribui para aumentar a quantidade de ADMA no citoplasma das células endoteliais, o que prejudica a síntese de óxido nítrico de maneira dose-dependente, com todas as consequiências para a fisiologia endotelial que a ausência desse importante vasodilatador traz. Acredita-se que a homocisteína não afete a produção ribossômica de DDAH, o que foi constatado pela dosagem enzimática com anticorpos monoclonais contra DDAH-1, mas não pôde ser feito em relação à DDAH-2, por ainda não existir disponibilidade de anticorpos monoclonais específicos contra essa enzima (24).

Leoncini, Pascale e Signorello publicaram resultados que indicam na mesma direção, mostrando que a homocisteína inibe o transporte de L-arginina de maneira dose-dependente, além de aumentar a reatividade plaquetária á trombina (25).

O mesmo grupo de autores investigou, posteriormente, se o mesmo mecanismo era válido em humanos. Após uma carga de metionina, foram encontradas elevações nos níveis séricos de homocisteína e de ADMA e redução na capacidade de vasodilatação (26). Além disso, determinou-se correlação entre os níveis de homocisteína e de ADMA e entre os níveis de ADMA e a vasodilatação.

Como se sabe, pacientes com insuficiência renal crônica tendem a apresentar aterosclerose, e há estudos que indicam que esse 
efeito pode ocorrer independentemente de outros fatores de risco clássicos para doença cardiovascular, bastando, para tanto, a disfunção do endotélio vascular (27). Entre os mecanismos responsáveis por essa disfunção, sugere-se o acúmulo de ADMA nas células, causado não somente pela excreção ineficiente pela urina, como também em conseqüência do aumento da concentração sérica de homocisteína. Observase que a função vasodilatadora do endotélio pode ser ao menos parcialmente recuperada pela administração intravenosa de L-arginina, o precursor do NO, ou por hemodiálise, que remove a ADMA do sangue (24). Esse efeito foi comprovado in vitro por MacAllister e colaboradores, que observaram vasoconstrição em resposta à inibição farmacológica da DDAH e a reversão desse efeito após a adição de L-arginina ao meio (28). Já foi demonstrada a validade da administração de folato a crianças com insuficiência renal crônica, como forma de se preservar a função endotelial e atenuar a oxidação de LDL, reduzindo, assim, as chances de desenvolvimento de aterosclerose nesses pacientes (29).

Não há dúvidas de que a homocisteína aumenta a concentração intracelular de ADMA, ao inibir diretamente a atividade da DDAH, provavelmente ao oxidar grupos sulfidril críticos para essa enzima. Essa hipótese é reforçada pela ação protetora exercida pela adição de 100 micromol/L de ditiocarbamato de pirrolidina (PDTC), um tiol antioxidante que protege grupos sulfidril, ao meio. Comprovando-se o papel da ADMA como regulador endógeno da síntese de $\mathrm{NO}$, a compreensão de vários aspectos $\mathrm{da}$ fisiopatologia das doenças vasculares crescerá.
Também já foi comprovado que a LDL oxidada inibe a função da DDAH, ao criar estresse oxidativo (30) e é possível que a ADMA seja o mecanismo efetor responsável pela patogênese de vários dos fatores de risco clássicos para doenças cardiovasculares, como hipertensão, tabagismo, hipercolesterolemia e hiperglicemia, uma vez que pacientes com essas características apresentam níveis séricos elevados de ADMA (31).

\section{CONSIDERAÇÕES FINAIS}

Há fartas evidências que relacionam a homocisteína ao desenvolvimento de aterosclerose, sugerindo que a redução dos níveis séricos de homocisteína poderia ser valioso para evitar o desenvolvimento futuro de doença cardiovascular. Entretanto, o impacto clínico desse tipo de estratégia ainda precisa ser determinado por estudos clínicos de grande porte.

A compreensão da fisiologia do endotélio vascular ainda é muito limitada, não estando completamente disponível o conhecimento de todos os seus reais fatores regulatórios e deixando cada vez mais claro que são muitas as influências a interagirem e se equilibrarem para possibilitar o seu perfeito funcionamento. Estando todos esses fatores vulneráveis a inúmeros outros mecanismos regulatórios, abre-se um vasto campo de pesquisa clínica, laboratorial e genética, que certamente terá papel preponderante tanto no tratamento quando na prevenção das doenças cardivasculares, que são a maior causa de mortes no mundo moderno.

\section{Homocysteine as a risk factor for atherosclerosis}

\section{Abstract}

Determining risk factors for cardiovascular diseases is important not only to guide correctly preventive programs, but also to identify individuals under cardiovascular risk that do not fit into any of the conventional cardiovascular risk factors: diabetes, systemic hypertension, sedentarism and smoking. In recent years, homocysteine has received great attention of cientists and several studies suggest that it may be an important cardiovascular risk factor, although some controversies remain. This paper aims at reviewig the association between homocysteine blood levels and the development of atherosclerosis, and the mechanisms by which such association manifests itself. Based upon the articles reviewed, one realizes that homocysteine blood levels 
have been frequently associated to the development of atherosclerosis and that, recently, enough evidence has been found to suggest this relation to be causal, direct and independent of the action of other factors. However, the clinical impact of approaches targeting a decrease in homocysteine blood leves remais to be determined by large clinical trials.

Keywords: homocysteine; atherosclerosis; cardiovascular disease.

\section{REFERÊNCIAS}

$1 \mathrm{CHOY}$, P. C. et al. Atherosclerosis risk factors: the possible role of homocysteine. Mol. Cell. Biochem., The Hague, v.207, p.143-148, 2000 .

2 HANKEY, G.J.; EIKELBOOM, J.W. Homocysteine and vascular disease. Lancet, London, v.354, p.407-413, 1999.

3 FALLEST-STROBL, P.C. et al. Homocysteine: a new risk factor for atherosclerosis. Am. Fam. Physician, Kansas City, v.56, p.1607-1612, 1997.

4 WELCH, G.N.; LOSCALZO, J. Mechanisms of disease: homocysteine and atherothrombosis. N. Engl. J. Med., Boston, v.338, p.1042-1050, 1998.

5 ZHLOBA, A.A.; BLASHKO, E.L. Liquid chromatographic determination of total homocysteine in blood plasma with photometric detection. J. Chromatogr. B. Analyt. Technol. Biomed. Life Sci., Amsterdam, v.800, p.275280, 2004.

6 EIKELBOOM, J.W. et al. Homocyst(e)ine and cardiovascular disease: a critical review of the epidemiologic evidence. Ann. Intern. Med., Philadelphia, v.131, p.363-375, 1999.

7 KANG, S.S.; WONG, P.W.; MALINOW, M.R. Hyperhomocyst(e)inemia as risk factor for occlusive vascular disease. Annu. Rev. Nutr., Palo Alto, v.12, p.279-298, 1992.

8 SANTARELLI, L. et al. Atrophic gastritis as a cause of hyperhomocysteinaemia. Aliment. Pharmacol. Ther., Oxford, v.19, p.107-111, 2004.
9 GUTHIKONDA, S.; HAYNS, W.G. Homocysteine as a novel risk factor for atherosclerosis. Curr. Opin. Cardiol., Philadelphia, v.14, p.283-291, 1999.

10 McCULLY, K.S. Vascular pathology of homocysteinemia: implications for the pathogenesis of arteriosclerosis. Am. J. Pathol., Bethesda, v.56, p.111-128, 1969.

11 NYGARD, O. et al. Total plasma homocysteine and cardiovascular risk profile: the Hordaland homocysteine study. JAMA: The Journal of the American Medical Association, Chicago, v.274, p.1526-1533, 1995.

12 CLARKE, R. et al. Hyperhomocysteinemia: an independent risk factor for vascular disease. N. Engl. J. Med., Boston, v.324, p.1149-1155, 1991.

13 GEISEL, J. et al. The impact of hyperhomocysteinemia as a cardiovascular risk factor in the prediction of coronary disease. Clin. Chem. Lab. Med., Berlin, v.41, p.15131517, 2003.

14 VAN DEN BOSCH, M.A. et al. Hyperhomocysteinemia and risk for peripheral arterial occlusive disease in young women. J. Vasc. Surg., St. Louis, v.38, p.772-778, 2003. 15 SPARK, J.I.; LAWS, P.; FITRIDGE, R. The incidence of hyperhomocisteinaemia in vascular patients. Eur. J. Vasc. Endovasc. Surg., London, v.26, p.558-561, 2003.

16 BOUSHEY, C.J. et al. A quantitative assessment of plasma homocysteine as a risk factor for vascular disease. JAMA: The Journal of the 
American Medical Association, Chicago, v.274, p.1049-1057, 1995.

17 BUEMI, M. et al. Effects of homocysteine on proliferation, necrosis, and apoptosis of vascular smooth muscle cells in culture and influence of folic acid. Thromb. Res., Elmsford, v.104, p.207-213, 2001.

18 CHAMBERS, J.C. et al. Plasma homocysteine concentrations and risk of coronary heart disease in UK and Indian, Asian and European men. Lancet, London, v.355,p.523-527, 2000.

19 STABLER, S.P. et al. Elevation of total homocysteine in the serum of patients with cobalamin or folate deficiency detected by capillary gas chromatography-mass spectrometry. J. Clin. Invest., Thorofare, v.81, p.466-474, 1988.

20 PIETRZIK, K.; BRÖNSTRUP, A. The role of homocysteine, folate and other B-vitamins in the development of atherosclerosis. Arch. Latinoam. Nutr., Caracas, v.47, p.9-12, 1997.

21 VERMEULEN, E.G.J. et al. Effect of homocysteine-lowering treatment with folic acid plus vitamin $B_{6}$ on progression of subclinical atherosclerosis: a randomized, placebocontrolled trial. Lancet, London, v.355, p.517522, 2000.

22 CHEN, C. et al. Homocysteine decreases endothelium-dependent vasorelaxation in porcine arteries. J. Surg. Res., New York, v.102, p.22-30, 2002.

23 LANG, D. et al. Homocysteine-induced inhibition of endothelium-dependent relaxation in rabbit aorta: role for superoxide anions. Arterioscler. Thromb. Vasc. Biol., Baltimore, v.20, p.42, 2000.
24 STÜHLINGER, M.C. et al. Homocysteine impairs the nitric oxide synthase pathway: role of asymmetric dimethylarginine. Circulation, Hagerstown, v.104, p.2569-2575, 2001.

25 LEONCINI, G.; PASCALE, R.; SIGNORELLO, M.G. Effects of homocysteine on L-arginine transport and nitric oxide formation in humman platelets. Eur. J. Clin. Investigation, Oxford, v.33, p.713-719, 2003. 26 STÜHLINGER, M.C. et al. Endothelial dysfunction induced by hyperhomocyst(e)inemia: role of asymmetric dimethylarginine. Circulation, Hagerstown, v.108, p.933-938, 2003.

27 KARI, J.A. et al. Physiology and biochemistry of endotelial function in children with chronic renal failure. Kidney Int., Malden, v.52, p.468-472, 1997.

28 MACALLISTER, R.J. et al. Regulation of nitric oxide synthesis by dimethylarginine demethylaminohydrolase. Br. J. Pharmacol., London, v.119, p.1533-1540, 1996.

29 BENNETT-RICHARDS, K. et al. Does oral folic acid lower homocysteine levels and improve endothelial functions in children with chronic renal failure? Circulation, Hagerstown, v.105, p.1810-1815, 2002.

30 ITO, A. et al. Novel mechanism for endothelial disfunction: dysregulation of dimethylarginine-dimethylaminohydrolase. Circulation, Hagerstown, v.99, p.3092-3095, 1999.

31 COOKE, J.P. Does ADMA cause endothelial dysfunction? Arterioscler. Thromb. Vasc. Biol., Baltimore, v.20, p.2032-2037, 2000. 